\title{
OCR-independent and Segmentation-free Word- Spotting in Handwritten Arabic Archive Documents
}

\author{
Aouadi N. \\ LaTICE, Research Laboratory of Technology of \\ Information and Communication \& Electrical Engineering \\ 5, Avenue Taha Hussein, BP 56 Bab Mnara, \\ Tunis, Tunisia \\ nabil.aouadi@utic.rnu.tn
}

\author{
Kacem A. \\ LaTICE, Research Laboratory of Technology of \\ Information and Communication \& Electrical Engineering. \\ 5, Avenue Taha Hussein, BP 56 Bab Mnara, \\ Tunis, Tunisia \\ Afef.kacem@esstt.rnu.tn
}

\begin{abstract}
In this paper, a word-spotting approach is presented that can help in reading handwritten Arabic Archive Documents. Because of the low quality of these documents, the proposed approach is free segmentation, independent of OCR, using a global transformation of word images. It is a based learning approach which employs Generalized Hough Transform (GHT) technique. It detects words, described by their models, in documents images by finding the model's position in the image. With the GHT, the problem of finding the model's position is transformed to a problem of finding the transformation's parameter that maps the model into the image. Parameters such as Hough threshold and distance between voting points are considered for a better location and recognition of words. We tested our system on registers from the 19th century onwards, held in the National Archives of Tunisia. Our first experiments reach an average of $94 \%$ of well-spotted words.
\end{abstract}

Keywords- OCR, Word-spotting, Generalized Hough Transform, Clustering, Handwritten Recognition, Historical document.

\section{INTRODUCTION}

A large number of historical documents are kept in the Tunisian National Archives; so having digital versions of old and fragile documents is a good way of preserving them and it makes them useful for several studies and projects. But they are often large, unstructured, and only available in image formats, which makes them difficult to access. In particular, finding specific locations of interest in a handwritten image collection is generally very tedious. Easy access to such collections requires an index, which is currently created manually at great cost in terms of time and money.

This paper explores the use of the Generalized Hough Transform (GHT) technique to spot handwriting word images. The proposed system can be applied for Arabic historical manuscripts retrieval without recourse to any OCR or previous block or word segmentation steps. The system, described here, has been built on a subset of the National Archives of Tunisia collection. We are interested by documents that are from the 19th century which correspond to enumeration registers at the colonial period of Tunisia. Figure 1 displays a small portion of this manuscript.

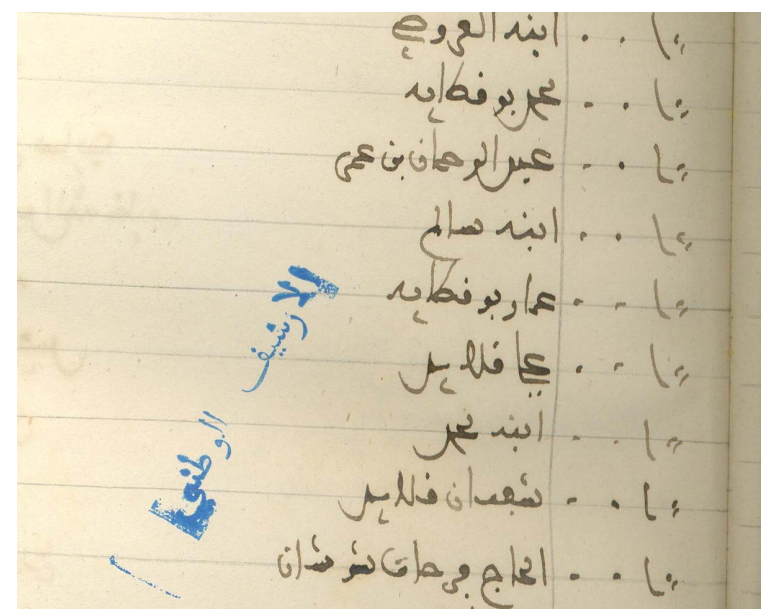

Fig. 1. Portion of Archive Register Page

Actually, there are works devoted to the word-spotting. A detailed survey on the word-spotting and information retrieval from document images can be found in [1, 2]. The first approach to the word-spotting was the use of index of descriptors for each document provided manually by experts. Then, with the improvement in character recognition field, OCR packages were applied to documents in order to convert them to text [3-28]. As OCR accuracy requirements for information retrieval are considerably low, methods with the ability of tolerating errors of OCR have, also been developed [4]. Lately, thanks to improvement in document image processing field, techniques that make use of images instead of OCR were also introduced. In this direction, we can mention two major approaches: segmentation-based or segmentationfree approaches. In the first approach, there is a great effort to solve the word segmentation problem [5-8]. In the second approach, the query word image is fitted to the corresponding word images in the document without any segmentation involved, mostly seen the underlying problem as a template matching.

The template based methods [8, 12-20] match a query word image with labeled keyword template images. An advantage of these methods is that template images are rather easy to obtain, even if the underlying language and its alphabet are unknown. However, such systems are limited by the fact that for each 
possible keyword that is to be spotted, at least one template image is needed and unknown out-of-vocabulary words cannot be spotted at all. Also, they typically have a low generalization capability to unknown writing styles. For a better generalization capability, learning-based methods $[9,21-26]$ are proposed. They employ statistical learning methods to train a keyword model that is then used to score query images.

Some mentioned works can give very good results to the specific applications they have been built for. But, some of them require extensive manual work and can only process predefined keywords, while many of them require extensive training. Some of related works are summarized in Table I.

TABLE I. RESULTS FOR SOME PREVIOUS WORKS

\begin{tabular}{|c|c|c|c|c|}
\hline Author & Method & 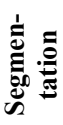 & Nature/Script & 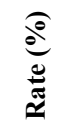 \\
\hline $\begin{array}{l}{[2] \mathrm{A} .} \\
\text { Fischer }\end{array}$ & $\begin{array}{l}\text {-Character Hidden } \\
\text { Markov Model }\end{array}$ & 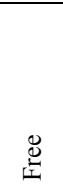 & $\begin{array}{l}\text {-English Handwritten } \\
\text {-Photography text } \\
\text { image in Parzival } \\
\text { database }\end{array}$ & 88 \\
\hline $\begin{array}{l}{[8]} \\
\text { A. } \\
\text { Bhardwaj }\end{array}$ & $\begin{array}{l}\text {-Similarity technique } \\
\text {-Moment } \quad \text { Based } \\
\text { Features }\end{array}$ & 苋 & $\begin{array}{l}\text { - English handwritten } \\
\text { - Hindi handwritten } \\
\text { - Sanskrit handwritten }\end{array}$ & $\begin{array}{l}66 \\
71 \\
88\end{array}$ \\
\hline $\begin{array}{l}{[18]} \\
\text { T. } \\
\text { Adawek }\end{array}$ & $\begin{array}{l}\text { - Holistic word } \\
\text { recognition } \\
\text {-Matching word } \\
\text { contour }\end{array}$ & 总 & $\begin{array}{l}\text {-Latin handwritten } \\
\text { historical document }\end{array}$ & 83 \\
\hline $\begin{array}{l}{[20]} \\
\text { K. } \\
\text { Terasawa }\end{array}$ & $\begin{array}{l}\text {-Line segmentation } \\
\text {-Continuous dynamic } \\
\text { programming } \\
\text {-Gradient distribution }\end{array}$ & 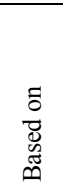 & $\begin{array}{l}\text {-Manuscripts English } \\
\text {-Manuscript Japanese }\end{array}$ & 97 \\
\hline \begin{tabular}{|l}
{$[7]$} \\
T. \\
Konidaris
\end{tabular} & $\begin{array}{l}\text {-Syntetic data } \\
\text {-User feedback }\end{array}$ & 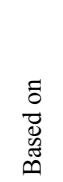 & $\begin{array}{l}\text {-Historical Printed } \\
\text { Document }\end{array}$ & 80 \\
\hline $\begin{array}{l}{[6] \quad \text { A. }} \\
\text { Balasubra } \\
\text { manian }\end{array}$ & $\begin{array}{l}\text {-Based Matching } \\
\text {-Image features } \\
\text {-Word-level } \\
\text {-DTW based partial } \\
\text { machine }\end{array}$ & 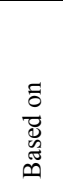 & $\begin{array}{l}\text {-English Printed } \\
\text { document images } \\
\text {-Hindi Printed } \\
\text { document images }\end{array}$ & $\begin{array}{r}97,7 \\
93,7\end{array}$ \\
\hline
\end{tabular}

In this work, we propose an OCR-independent and freesegmentation approach, in which words can be spotted without pre-segmenting text-lines into words. It is also a learning-based approach. It relies on a global transform of the words using GHT and clustering technique. The main advantage of GHT is that it is relatively unaffected by noise. It is known for its capacity to detect objects in a given image depending on its contextual position (at the beginning, in the middle, at the end or isolated). In our study, we focused on the generalized Hough Transform developed by Ballard [29] which is a standard technique in computer vision having many applications for detecting lines, circles, and any arbitrary shapes as shown in figure 2. It is robust to partial or slightly deformed words and to the presence of additional structures in the image (i.e., other lines, curves, etc.). It can find multiple occurrences of a word during the same processing pass. A known disadvantage of the proposed approach is, however, that it requires a set of transcribed word images for training that may be costly to obtain, in particular for historical documents.

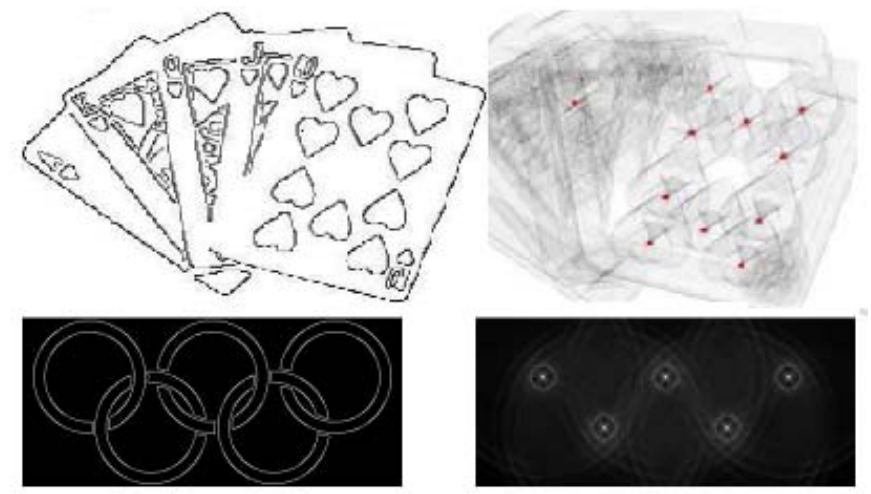

Fig. 2. Capabilities of GHT to detect any arbitrary form

The remainder of the paper will be structured as follows. In Section 2, characteristics of used documents are presented. In Section 3, the proposed system is detailed. In Section 4, the evaluation results on representative historical registers are given, and in Section 5, conclusions are drawn.

\section{USED DOCUMENTS}

As illustrated in figure 1, used documents are old, noisy and high degraded. They consist of text-lines; each of them is composed of a list of words: personal names. Text-lines are of different length. These documents are written, by a single author who used line support, and in Arabic using old scripts.

Due to the style of the writing, attachment occurred between the words of the successive text-lines and vertical and/or horizontal ligatures are easily introduced between the parts of words. Note that images of multiple instances of the same word are likely to look similar. But some letters changed the shape. For example, the letter 'ي can be flattened and oriented right to left. Furthermore, by using Arabic old scripts, some letters have also changed the number and/or position of their diacritic points. For example, the letter ' $ق$ ' is written with a single diacritic point above the letter body instead of two points and the letter ' $i$ ' is written with a single diacritic point below the letter body. The register has the following characteristics:

- Hiding or loss of structural information (multiple fallback, collages, creased or torn pages)

- Different length of lines, more or less fluctuating. 
- Interleaving lines, overlapping of connected components as shown in figure 3.

- Horizontal and vertical ligatures as shown in figure 4.

- $\quad$ Some letters changed the shape as shown in figure 5.

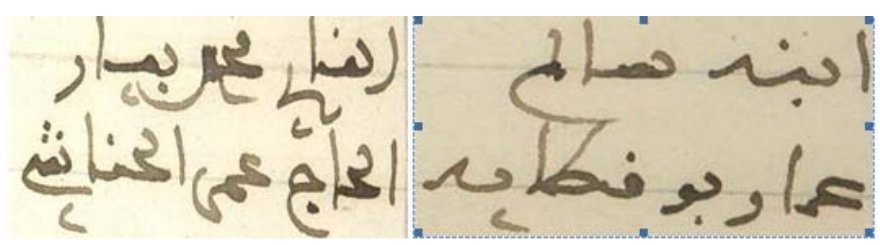

Fig. 3. Examples of overlapping lines.

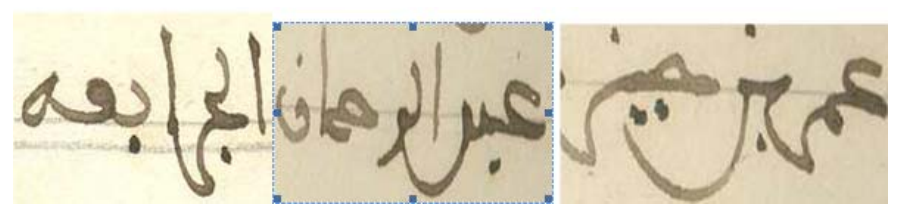

Fig. 4. Words with horizontal and vertical ligatures.

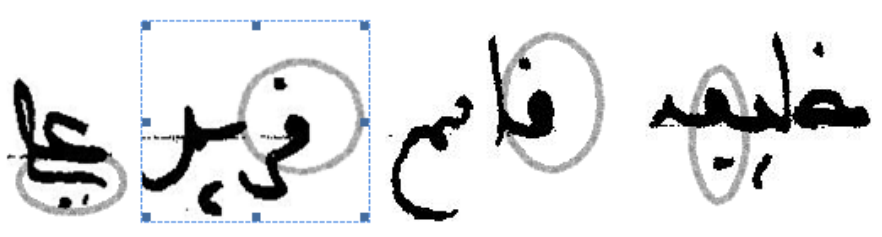

Fig. 5.Old Arabic Writing.

Because of the low quality of these documents (background noise, artifacts due to aging, interfering lines), the complexity of Arabic handwriting and OCR failure, searching and indexing these documents are a very challenging problem. We propose an independent-OCR and segmentation-free system that can be used to spot handwritten word images as it will be explained next.

\section{OVERVIEW OF THE PROPOSED SYSTEM}

Our system retrieves handwritten pages given word queries. It is based on GHT and clustering technique. The system matches an input text word query with one or multiples word models, stored in a dictionary, to determine the associate word query model. Using the Hough parameters of the word query model and a distance that might indicate a correspondence, the image word query will be located and spotted in the document image as illustrated in Figure 6. To better understand how to spot words using GHT, we will give a brief explanation of this technique; then, we will explain how it is possible to detect and locate words.

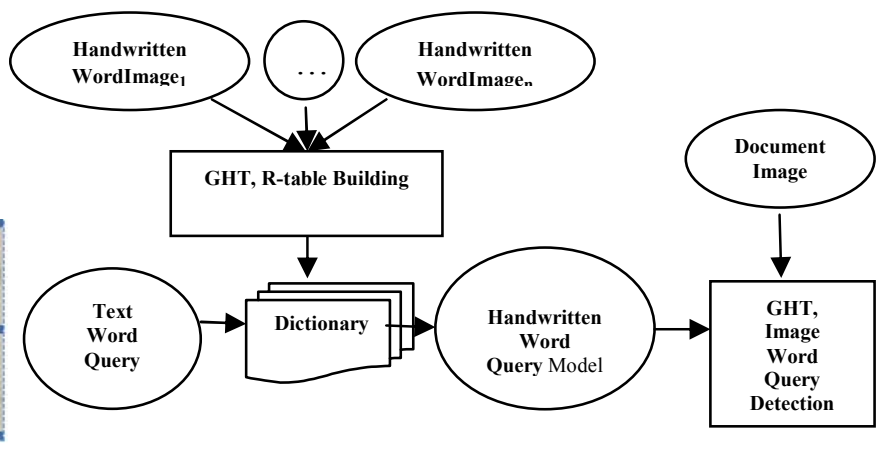

Fig. 6. Proposed System Overview.

\section{A. Generalized Hough Transform (GHT)}

The classical Hough Transform has been generalized so that it is capable of detecting arbitrary curved shapes. In case where the shape we wish to isolate does not have a simple analytic equation describing its boundary, we can still use a generalized form of the Hough transform. The essential idea is that, instead of using the parametric equation of the curve, we use a look-up table to define the relationship between the boundaries coordinates and orientation, and the Hough parameters [11]. Obviously, the look-up table values must be computed during a training phase using a prototype shape.

Suppose we know the shape and orientation of the required object (See Figure 7), the first step is to select an arbitrary reference point $\left(\mathrm{X}_{\text {ref }}, \mathrm{Y}_{\text {ref }}\right)$ in the object. We now define the shape in terms of the distance and angle of lines from the boundary to this reference point. For all points of the boundary, we draw a line to the reference point. We then compute the orientation of the boundary, $\Omega_{\mathrm{i}}$ say, and make a note in the look-up table of the distance and direction from the boundary point to the reference point at a location in the look-up table indexed by the boundary orientation $\Omega_{\mathrm{i}}$. Since it is probable that there will be more than one occurrence of a particular orientation as we travel around the boundary, we have to make provision for more than one pair of distance and angle values. This look-up table is called R-table.

The Hough transform space is now defined in terms of the possible positions of the shape in the image, i.e. the possible ranges of $X_{\text {ref }}$ and $Y_{\text {ref. }}$ To perform the transform on an image we compute the point $\left(\mathrm{X}_{\text {ref }}, \mathrm{Y}_{\text {ref }}\right)$ from the coordinates of the boundary point, the distance $r$ and the angle $\beta$ :

$$
\begin{aligned}
& \mathrm{X}_{r e f}=x+r \cos (\beta) \\
& Y_{r e f}=y+r \sin (\beta)
\end{aligned}
$$

The values of $r$ and $\beta$ are derived from the R-table by computing the boundary orientation $\Omega$ at that point and using it as an index to the $\mathrm{R}$-table, reading off all the $(\mathrm{r}, \beta)$ pairs. The accumulator array cell $\left(\mathrm{X}_{\text {ref }}, \mathrm{Y}_{\text {ref }}\right)$ is then incremented. We reiterate this process for all edge points in the image. We infer the presence of the shape by identifying local maxima in the accumulator array. 


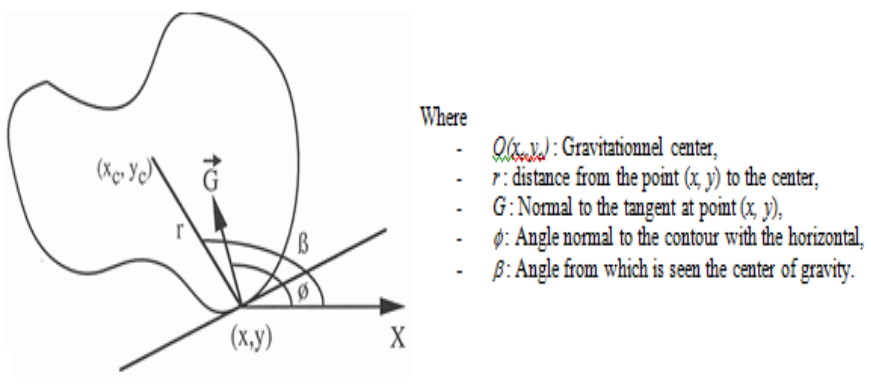

Fig. 7. Object model

\section{B. Word-spotting by GHT}

Our system finds Arabic manuscripts words dealing only with pseudo-words for two reasons: 1) Apply GHT in a portion of a word or a pseudo-word is simpler and faster than apply on a whole word and 2) many words have similar portion as shown in Figure 8.
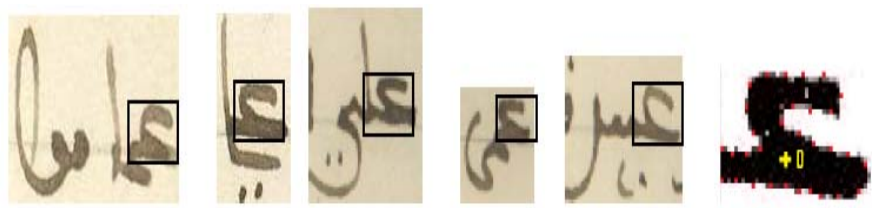

Fig 8. Same pseudo-word belonging to different names.

Thus, we proceed by manual segmentation of queries words in pseudo-words for the modeling during the training phase of the system.

To spot words, our system has to go through a training phase where the objective is to generate models of words or parts of words considering different samples, and then build the corresponding R-tables. To this end, the system follows the next steps, for each word or part of word:

- Extract the boundary:

- Define a reference point $\left(\mathrm{x}_{\mathrm{c}}, \mathrm{y}_{\mathrm{c}}\right)$ somewhere inside the shape (e.g., the gravitational center). For each point ( $x$, $y$ ) on the boundary, find two parameters: $r$ and $\beta$, using the next equations, and the gradient direction $\mathrm{G}$

$$
\begin{aligned}
& r=\sqrt{\left(\mathrm{x}-\mathrm{x}_{\mathrm{c}}\right)^{2}+\left(\mathrm{y}-\mathrm{y}_{\mathrm{c}}\right)^{2}} \\
& \beta=\tan ^{-1}\left(\left(\mathrm{x}-\mathrm{x}_{\mathrm{c}}\right) /\left(\mathrm{y}-\mathrm{y}_{\mathrm{c}}\right)\right)
\end{aligned}
$$

- Add the pair (r, $\beta$ ) to the table entry with its closest to G. The R-table allows, using the boundary points and gradient angle, to re-compute the location of the reference point. Notice that we need to build a separate R-table for each different part of word.

- Prepare a 2D Hough array $\mathrm{H}\left(\mathrm{x}_{\mathrm{c}}, \mathrm{y}_{\mathrm{c}}\right)$ initialized to 0 .

After that, the system builds a dictionary of word models (see Table II). For each of them, the system associates the corresponding d-cluster (the minimal distance between word clusters which is empirically determined), the parts of word that compose it, and for each part of word, its relative R-table and a Hough-threshold (number of minimal votes for the part of word).

\begin{tabular}{|c|c|c|c|c|}
\hline \multirow{2}{*}{ Word } & \multicolumn{4}{|c|}{ R-tables } \\
\hline & \multicolumn{2}{|c|}{ Word Parts } & d-cluster & s-Hough \\
\hline \multirow{8}{*}{ Mot1 } & \multirow{4}{*}{1} & $\left(\beta_{11}, r_{11}\right)$ & \multirow{4}{*}{ d1 } & \multirow{4}{*}{ s1 } \\
\hline & & $\left(\beta_{12}, \mathrm{r}_{12}\right)$ & & \\
\hline & & $\left(\beta_{13}, \mathrm{r}_{13}\right)$ & & \\
\hline & & $\left(\beta_{14}, \mathrm{r}_{14}\right)$ & & \\
\hline & \multirow{4}{*}{2} & $\left(\beta_{21}, \mathrm{r}_{21}\right)$ & \multirow{4}{*}{$\mathrm{d} 2$} & \multirow{4}{*}{ s2 } \\
\hline & & $\frac{\ldots \ldots}{(\beta}$ & & \\
\hline & & $\frac{\left(\beta_{2, \mathrm{k}}, \mathrm{r}_{11}\right)}{\left(\beta_{2, \mathrm{k}+1}, \mathrm{r}_{11}\right)}$ & & \\
\hline & & $\left(\beta_{2, \mathrm{k}+\mathrm{n}}, \mathrm{r}_{11}\right)$ & & \\
\hline \multirow{2}{*}{ علي } & $\varepsilon$ & $\ldots$ & \multirow[b]{2}{*}{60} & \multirow[b]{2}{*}{16} \\
\hline & لي & $\ldots$ & & \\
\hline \multirow{2}{*}{ محمد } & مد & $\ldots$ & \multirow{2}{*}{40} & \multirow{2}{*}{17} \\
\hline & مد & $\ldots$ & & \\
\hline
\end{tabular}

TABLE II. PORTION OF THE BUILT DICTIONARY

\section{Algorithm GHT}

- Initialize an array called accumulator $\mathrm{A}\left[\mathrm{x}_{\min . .} . \mathrm{x}_{\max }, \mathrm{y}_{\min . .} \mathrm{y}_{\max }\right]$

- For each contour point $\mathrm{P}(\mathrm{x}, \mathrm{y})$, calculate the angle $\mathrm{b}$ witch provide input $(b=)$ in R-table

- For each pair of line in R-table

- Compute $x_{0}=x+r \cos (\beta), y_{0}=y+r \sin (\beta)$

- Increment $\mathrm{A}\left[\mathrm{x}_{0}, \mathrm{y}_{0}\right]$

- Determine the pair $(\mathrm{x}, \mathrm{y})$ that maximizes $\mathrm{A}[\mathrm{x}, \mathrm{y}]$

Now, giving a text query word, the system first consults the dictionary in search of the appropriate models of the word parts. Then, for each found word part model, the system routes the register page image and looks for the voting points. If the number of the voting points exceeds a fixed Hough-threshold, then a voting cluster will be formed. Thus, the system produces the voting clusters for the different parts of the query word. If the distance between the voting clusters is less than a distance d-cluster, then these clusters will be merged into one cluster and the query image word will be spotted.

Let us find all positions of the word " عمر" in the document image. Notice that, our system has already stored, in the dictionary at the training stage, this entry, manually segmented into two parts: " $s "$ and "م" (See Figure 9). Each word part is described by its R-table. A Hough-threshold and d-cluster are respectively fixed to 16 and 60 pixels after empirical tests. In Figure 10, the two clusters which respectively vote for " $s$ " and "م" are merged into one because the distance between them is less than 60 pixels.
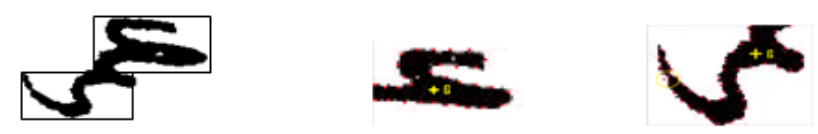

Fig. 9. Parts of the word عر 

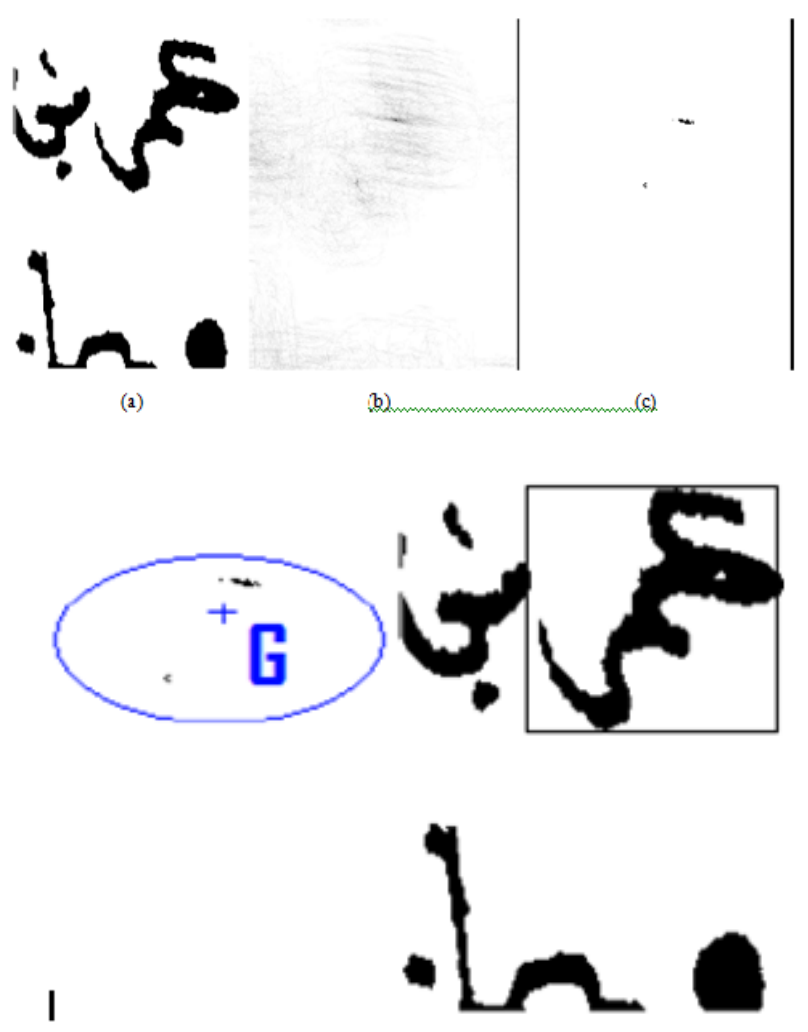

(d)

(e)

Fig. 10. (a) Image space, (b) Hough space, (c) voting points selection of $\mathbf{s}$ and $\mathbf{s}$ 'parts, (d) Cluster merging (e) $\boldsymbol{乛}_{\text {Word-spotting. }}$

Figure 11 displays obtained results from word-spotting of the words عماموا and ابنه (in the first text-line of the figure 6(a)) has been confused with عماموا because they look similar. More results are given in Table III.
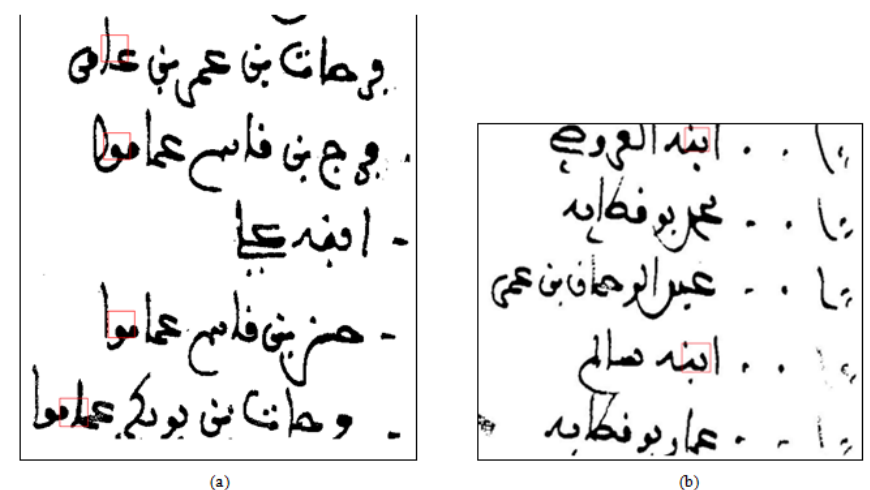

Fig. 11. (a) Word-spotting of the word lase, (b) Word-spotting of the word / انه

Experiments have been conducted on a archive documents composed of 23 pages. Each page consists of about 36 lines. Each text-line contains 4 words on average. So we handle a total of 3312 words. The obtained results are quite satisfactory given the specificities of these documents. Over $94 \%$ of words are well spotted. This approach is unaffected by image noise. But the main drawbacks of this approach, based on GHT, are its substantial computational and storage requirements that become acute when word orientation and scale have to be considered.

TABLE III. SOME OBTAINED RESULTS

\begin{tabular}{|c|c|c|c|c|}
\hline Word & Samples & $\begin{array}{c}\text { Test } \\
\text { samples }\end{array}$ & $\begin{array}{c}\text { Hough } \\
\text { treshold }\end{array}$ & $\begin{array}{l}\text { Recogniti- } \\
\text { on Rate }\end{array}$ \\
\hline & 60 & 5 & 15 & $91 \%$ \\
\hline & 25 & 4 & 13 & $92 \%$ \\
\hline & 45 & 5 & 10 & $88 \%$ \\
\hline & 15 & 3 & 12 & $97 \%$ \\
\hline & 15 & 3 & 12 & $93 \%$ \\
\hline & 30 & 7 & 14 & $96 \%$ \\
\hline & 50 & 12 & 16 & $98 \%$ \\
\hline & 30 & 8 & 15 & $95 \%$ \\
\hline
\end{tabular}

\section{CONCLUSION}

In this paper, a system appropriate for word-spotting in historical Arabic handwritten documents that does not require page segmentation was presented. It is based on GHT, which allows effective retrieval of words without using any OCR. The proposed system relies on two components: training and word detection phases for text word query resolution. The training phase is run only once for each query word and it consists of extracting image features from word images. More specifically, word image space is converted into parameter space using GHT. These parameters are later stored in R-tables. The final output of the training phase is a dictionary which includes, for each text query word, the R-tables relative to its image word parts, the d-cluster and Hough-threshold. In the query resolution phase, a text query word is looked up in the dictionary to extract part word models, and then by scanning document images, the system identifies all possible locations of the query word. Notice that GHT serves, here, not to only detect or locate words but also to recognize them. How to choose models to optimize GHT? How to enhance system robustness against inclination and image resolution? There is still lot to do but it is really worthwhile. 


\section{REFERENCES}

[1] D. Doermann, "The indexing and Retrieval of Document Images: A Survey", In computer Vision and Image Understanding, 70(3), pp. 287298, 1998.

[2] A. Fischer, A. Keller, V. Frinken, H. Bunke, "Lexicon-Free Handwritten Word-spotting Using Character HMMs", University of Bern, Pattern Recognition Letters, volume 33, Issue 7, pp. 934- 942, May 2012.

[3] J. Edwards, Y. W. Teh, D. Forsyth, R. Bock, M. Marie, G. Vesom, "Making Latin manuscripts searchable using gHMM's", In Conf. on neural information Processing Systems, Cambridge, USA, pp. 385-392, 2004.

[4] Y. Ishitani, "Model-based Information extraction Method tolerant of OCR Errors for Document images”, In ICDAR, Seattle, USA, , pp. 908915, 2001.

[5] T. M. Rath, , R. Manmatha, "Features for word-spotting in historical documents", In ICDAR, pp 218-222, 2007.

[6] A. Balasubramanian, M. Meshesha, C. V. Jawahar, "Retrieval from Document Image Collections”, In DAS, pp 1-12, 2006.

[7] T. Konidaris, B.Gatos, K. Ntkios, I. Pratikakis, S. Theodoridis, S. Perantonis, "Keyword-guided Word-spotting in Historical Printed Documents using Synthetic Data and User feedback". In IJDAR, vol. 9, pp 167-77, 2007.

[8] A. Bhardwaj, D, Jose, V. Govindaraju, "Script Independent Wordspotting in Multilingual Documents", In 2th International Workshop on Cross Lingual Information Access, pp 48-54, 2008.

[9] J. Rodriguez, F. Perronnin, Handwritten Word-Spotting Using Hidden Markov Models and Universal Vocabularies, Pattern Recognition 42 (9), pp. 2106-2116, 2009.

[10] http://fourier.eng.hmc.edu/e161/lectures/hough/node7.html.

[11] R. O. Duda, P. E. Hart, "Use of the Hough Transformation to Detect Lines and Curves in Pictures", Comm. ACM, Vol. 15, pp. 11 - 15, 1972.

[12] M 12. R. Manmatha, C. Han, E. Riseman, "Word-spotting: A New Approach to Indexing Handwriting", in: Proc. Int. Conf. on Computer Vision and Pattern Recognition, pp. 631-637, 1996.

[13] B. Zhang, S. N. Srihari, C. Huang, "Word Image Retrieval Using Binary Features", in: Proc. Document Recognition and Retrieval XI, vol. 5296, pp. 45-53, 2004.

[14] J. L. Rothfeder, S. Feng, T. M. Rath, "Using Corner Feature Correspondences to Rank Word Images by Similarity", in: Proc. Workshop on Document Image Analysis and Retrieval, pp. 30-35, 2003.

[15] T. M. Rath, R. Manmatha, "Word Image Matching Using Dynamic Time Warping”, in: Proc. Int. Conf. on Computer Vision and Pattern Recognition, pp. 521-527, 2003

[16] Y. Leydier, A. Ouji, F. LeBourgeois, H. Emptoz, "Towards an Omnilingual Word Retrieval System for Ancient Manuscripts", Pattern Recognition 42 (9), pp. 2089-2105, 2009.

[17] T. M. Rath, R. Manmatha, "Word-spotting for historical documents", Int. Journal on Document Analysis and Recognition 9, pp. 139-152, 2007.

[18] T. Adamek, N. E. Connor, A. F. Smeaton, Word Matching Using Single Closed Contours for Indexing Historical Documents, Int. Journal on Document Analysis and Recognition 9 (2), pp.153-165, 2007.

[19] J. Rodriguez, F. Perronnin, "Local Gradient Histogram Features for Word-spotting in Unconstrained Handwritten Documents", in: Proc. 1st Int. Conf. on Frontiers in Handwriting Recognition, pp. 7-12, 2008.

[20] K. Terasawa, Y. Tanaka, "Slit Style HOG Features for Document Image Word-spotting", in: Proc. 10th Int. Conf. on Document Analysis and Recognition, vol. 1, pp. 116-120, 2009.

[21] C. Choisy, Dynamic Handwritten Keyword-spotting Based on the NSHPHMM, in: Proc. 9th Int. Conf. on Document Analysis and Recognition, pp. 242-246, 2007.

[22] F. Perronnin, J. Rodriguez-Serrano, Fisher Kernels for Handwritten Word-spotting, in: Proc. 10th Int. Conf. on Document Analysis and Recognition, vol. 1, pp. 106-110, 2009.
[23] J. Edwards, Y.W. Teh, D. Forsyth, R. Bock, M. Maire, G. Vesom,Making Latin Manuscripts Searchable Using gHMM's, in: Advances in Neural Information Processing Systems, pp. 385-392, 2004.

[24] J. Chan, C. Ziftci, D. Forsyth, Searching Off-line Arabic Documents, in: Proc. Int. Conf. on Computer Vision and Pattern Recognition, pp. 14551462, 2006

[25] A. El Yacoubi, M. Gilloux, J.-M. Bertille, A Statistical Approach for Phrase Location and Recognition within a Text Line: An Application to Street Name Recognition, IEEE Trans. PAMI 24 (2), pp. 172-188, 2002.

[26] S. Thomas, C. C., L. Heutte, T. Paquet, An Information Extraction Model for Unconstrained Handwritten Documents, in: Proc. 20th Int. Conf. on Pattern Recognition, pp. 3412-3415, 2010.

[27] J. Ruiz-Pinales, E. Lecolinet, "Cursive Handwriting using the Hough Transform and Neural nework", in proc. $15^{\text {th }}$ Int.Conf. on Pattern Recognition 2000.

[28] M. Fakir .M.Hassani, C. Sodeyama, "On the Recognition Of Arabic, Characters using Hough Transform Technique", Malausian Journal of Computer Science, Vol.13 No.2, December 2000,pp.39-47.

[29] D.H. Ballard, "Generalized the Hough Transform to Detect Arbitrary Shapes". 